\title{
Relationship between the immunohistological examination and fluorescence visualization of oral squamous cell carcinoma
}

\author{
KEISUKE SUGAHARA $^{1,2}$, YU KOYAMA ${ }^{1}$, MASAHIDE KOYACHI ${ }^{1}$, SATORU MATSUNAGA ${ }^{2,3}$, KENTO ODAKA ${ }^{4}$, \\ KEI KITAMURA $^{5}$, KEI NAKAJIMA ${ }^{6}$, KENICHI MATSUZAKA ${ }^{6}$, SHINICHI ABE $^{3}$ and AKIRA KATAKURA ${ }^{1}$ \\ ${ }^{1}$ Department of Oral Pathobiological Science and Surgery; ${ }^{2}$ Oral Health Science Center; \\ Departments of ${ }^{3}$ Anatomy, ${ }^{4}$ Oral and Maxillofacial Radiology, ${ }^{5}$ Histology and Developmental Biology and \\ ${ }^{6}$ Clinical Pathophysiology, Tokyo Dental College, Tokyo 101-0061, Japan
}

Received December 18, 2019; Accepted April 27, 2020

DOI: $10.3892 / \mathrm{ol} .2020 .11772$

\begin{abstract}
Disorders of the oral mucosa are considered easy to diagnose since they can be visualized and examined directly. A change in the color of the oral mucosa reflects histopathological changes and is an important diagnostic parameter. However, the subjective perception of color varies. To determine the extent of resection for oral mucosa conditions, it is necessary to digitize the color and perform objective assessments. In recent years, fluorescence visualization devices and analysis software that measure tissue luminance $G$ have been employed for the identification of oral mucosa diseases. Fluorescence visualization is presumably based on the decrease in epithelial flavin adenine dinucleotide content and luminance $G$ values due to the destruction of collagen cross-links [fluorescence visualization loss (FVL)]. However, cases with differences between luminance values and histopathological presentation exist. Therefore, additional factors may affect fluorescence visualization. The present study used a portable, non-contact oral mucosa fluorescence visualization device for luminance measurements in seven patients with oral squamous cell carcinoma. Furthermore, Picro-Sirius Red and immunohistochemical staining were performed for CK13, CK17, Ki67, p53 and E-cadherin in the FVL(+) (lesion) and FVL(-) (resection stump) areas to elucidate the principle of fluorescence visualization. Fluorescence was significantly lower in the FVL(+) than in the FVL(-) areas, and the mean luminance $G$ value was 56. The Picro-Sirius Red stain revealed collagen destruction in the FVL(+) areas but no collagen disruption in the FVL(-) areas. CK13 was negative in the FVL(+) and positive in the FVL(-) areas, whereas the opposite pattern was observed for CK17. In the FVL(+)
\end{abstract}

Correspondence to: Dr Keisuke Sugahara, Department of Oral Pathobiological Science and Surgery, Tokyo Dental College, 2-9-18 Kandamisaki-cho, Tokyo 101-0061, Japan

E-mail: ksugahara@tdc.ac.jp

Key words: fluorescence visualization, oral mucosal disease, oral squamous cell carcinoma, pathological approach area, p53 staining was positive. E-cadherin expression was enhanced in the FVL(-) areas and reduced in the FVL(+) areas. Furthermore, the luminance $G$ value tended to be lower in cases with weaker E-cadherin staining. The aforementioned results suggest that decreased E-cadherin expression may be a factor that regulates fluorescence visualization.

\section{Introduction}

The early diagnosis and treatment of oral squamous cell carcinoma (OSCC) significantly affects the prognosis and quality of life of patients. The change in the color of the mucous membrane is the most important diagnostic parameter for oral mucosa disorders, because it reflects histopathological alterations. However, the subjective perception of color varies even when different individuals simultaneously observe the same color. Because the human brain does not have the capacity to accurately reproduce information about color, it is difficult to objectively record color changes. The appropriate identification of color associated with an oral mucosa lesion for diagnostic purposes requires digitization of colors and their evaluation as objective data. The determination of the extent of excision of precancerous and OSCC lesions that should be undertaken is often done by vital staining with iodine solution (1); however, this approach also relies substantially on the subjective assessment made by technicians.

The relationship between reduced tissue autofluorescence and epithelial malformations has been reported in recent years with the advancements made in optical engineering. Therefore, fluorescence visualization devices have been widely used as auxiliary diagnostic tools and have contributed significantly to the diagnosis and treatment of pathological conditions of the oral mucosa. The diagnostic instrument, VELscope ${ }^{\circledR}$ (DenMat), used for the oral mucosa was developed in 2006; it uses the difference in visible light reflected by the mucous membrane and has been applied clinically (2-4). This device visualizes lesions via the irradiation of narrow-band blue light (400-460 nm) that enables light emission by collagen matrix or flavin adenine dinucleotide (FAD). Tumors and inflammatory processes are indicated by reduced FAD levels and fluorescence visualization loss (FVL) due to collagen cross-link (CCL) destruction. An oral mucosa fluorescence visualization 
device that uses an optical instrument and an analysis software calculating its $\mathrm{G}$ value has also been developed in Japan and has been examined for its usefulness in distinguishing lesions (5). However, there are cases characterized by differences between the luminance values and histopathological presentation, which have led to the hypothesis that a factor other than the described principle of fluorescence visualization exists.

In the present study, we aimed to elucidate the principle of fluorescence visualization. We used a portable, non-contact oral mucosa fluorescence visualization device to perform fluorescence visualization and obtain luminance measurements of pathologically altered oral mucosa; furthermore, we carried out Picro-Sirius Red staining of CCL and immunohistochemical staining for CK13, CK17, Ki67, p53, and E-cadherin.

\section{Materials and methods}

Subjects. Oral squamous cell carcinoma was fluorescently visualized and histopathologically assessed in seven patients examined at the Department of Oral Surgery, Tokyo Dental College, Suidobashi Hospital between April 2017 and August 2018. Patients with distant metastases at the time of clinical examination or those receiving preventive radiotherapy or chemotherapy were excluded from the study. This study was conducted with the approval of the Tokyo Dental College Ethics Committee (approval no. 759). Informed consent was obtained from all the participants. And this informed consent included consent to participate in the study and consent to publish their images. The patient characteristics are detailed in Table I.

Device. Fluorescent visualization of oral mucosa was conducted with IllumiScan ${ }^{\circledR}$ (SHOFU Inc.). It is a lightweight ( $\sim 320 \mathrm{~g})$, portable device that allows one-handed operation. IllumiScan ${ }^{\circledR}$ functions in a similar manner as an intraoral camera, capturing a fluorescent image of the observed intraoral site, saving it as digital data, and displaying the fluorescent image on a screen, which enables multiple observers to view it simultaneously (Fig. 1).

\section{Method for color measurements of fluorescence images}

Fluorescent visualization principle and assessment parameters. IllumiScan ${ }^{\circledR}$ enables the fluorescent visualization of oral mucosa through the observation of autofluorescence emitted by healthy mucosal cells. Fluorophores, such as the coenzyme FAD in the mucosal epithelium and CCLs in the stroma are excited by blue excitation light to generate bright green fluorescence. Cells with premalignant or malignant lesions or with abnormalities in the TCA cycle exhibit a decreased content of the above-mentioned epithelial or stromal fluorophores in comparison with healthy cells, which is consequently revealed as decreased fluorescence (a loss of fluorescence). More specifically, a loss of FAD fluorescence occurs in oral premalignant lesions, whereas in OSCC, a loss of FAD or CCL fluorescence is observed. Such fluorescence variations were, therefore, used for the objective assessment of oral mucosa in the present study.

Excitation light and fluorescence wavelength. Oral tissue fluoresces when illuminated with the excitation light emitted from IllumiScan ${ }^{\circledR}$. The horizontal axis in Fig. 2 indicates the wavelengths at which FAD and CCLs fluoresce, whereas the vertical axis shows the excitation wavelengths at which FAD and CCLs are excited to generate fluorescence. The range of these wavelengths was plotted as a circle and an ellipse for FAD and CCLs, respectively. A light gray zone in the figure indicates a band of the excitation wavelengths (peak: $425 \mathrm{~nm}$ ) at which the tissue was illuminated. FAD and CCLs were excited at wavelengths in the range where the circle (for FAD) or ellipse (for CCLs) areas overlap with the light gray zone; as a result, FAD and CCLs fluoresced at wavelengths in the range designated as a band of fluorescence-generated. The tissue was observed through the fluorescence filter of IllumiScan ${ }^{\circledR}$, which blocks the intense reflection of the excitation light (upper limit: $\sim 450 \mathrm{~nm}$ ). Therefore, we assessed fluorescence within a band of fluorescence-observable wavelengths shown as a gray zone in the figure, using the image analysis software described below (Fig. 2).

Analysis of fluorescence intensity using an image analysis software. The basic operation of our image analysis software is described below. An intraoral fluorescence image $(1,920 \times 1,080$ pixels) captured by the camera integrated in the IllumiScan ${ }^{\circledR}$ body was loaded onto the image analysis software on a personal computer (PC) to display the image downsized to $960 \times 540$ pixels. For each pixel of the image, RGB element values [a color representation with R (red), G (green), and $B$ (blue), each with values ranging from 0 to 255] were provided; the $G$ value indicated the intensity of the green color at an arbitrary point in the fluorescence image. The analysis software also provided: The ratio between two mean $G$ values obtained from selected regions in two different fluorescence images; an image showing gradients based on $\mathrm{G}$ values; selection of an area of concern (lesion area) to be analyzed; and the ratio between $G$ values inside and outside of the selected area. The gradient expressed in the present study was computed as follows: The mean $G$ value of a total of 25 pixels including a target pixel (5x5=25, a region-of-interest) in an intraoral fluorescence image on the $\mathrm{PC}$ was calculated and treated as a target intensity value in the region. Similarly, the mean $G$ value was calculated for each of the eight surrounding regions adjacent to the region-of-interest and treated as an adjacent intensity value for each surrounding region. The mean of absolute values of differences between the target intensity value and each of the adjacent intensity values was calculated and treated as a gradient value.

Method for the measurement and evaluation of $G$ values. The measured area was defined as a circle with a 30-pixel diameter. For subjects with an oral mucosa disease, $G$ values at three arbitrary points from a lesion area and a lesion-free area were measured, and the respective mean $G$ values were calculated for lesion and lesion-free areas. The ratio of the mean $G$ value from the lesion area versus that from the lesion-free area was also calculated. For healthy subjects, we measured $\mathrm{G}$ values in specific regions of oral mucosa, i.e., the tongue (lingual edge, sublingual surface, and dorsum of the tongue), the floor of the oral cavity, buccal mucosa, upper and lower lip mucosa, and maxillary and mandibular gingiva, excluding the lingual frenulum and median palatine suture because of abundant expression of keratinized 
Table I. Clinical information.

\begin{tabular}{cclll}
\hline Case & Age, years & Sex & Site & Type \\
\hline 1 & 62 & Male & Left tongue & Endophytic growth \\
2 & 43 & Female & Left tongue & Endophytic growth \\
3 & 59 & Male & Left tongue & Endophytic growth \\
4 & 73 & Male & Left tongue & Superficial growth \\
5 & 76 & Female & Left tongue & Superficial growth \\
6 & 66 & Male & Right tongue & Superficial growth \\
7 & 64 & Male & Left tongue & Endophytic growth \\
\hline
\end{tabular}
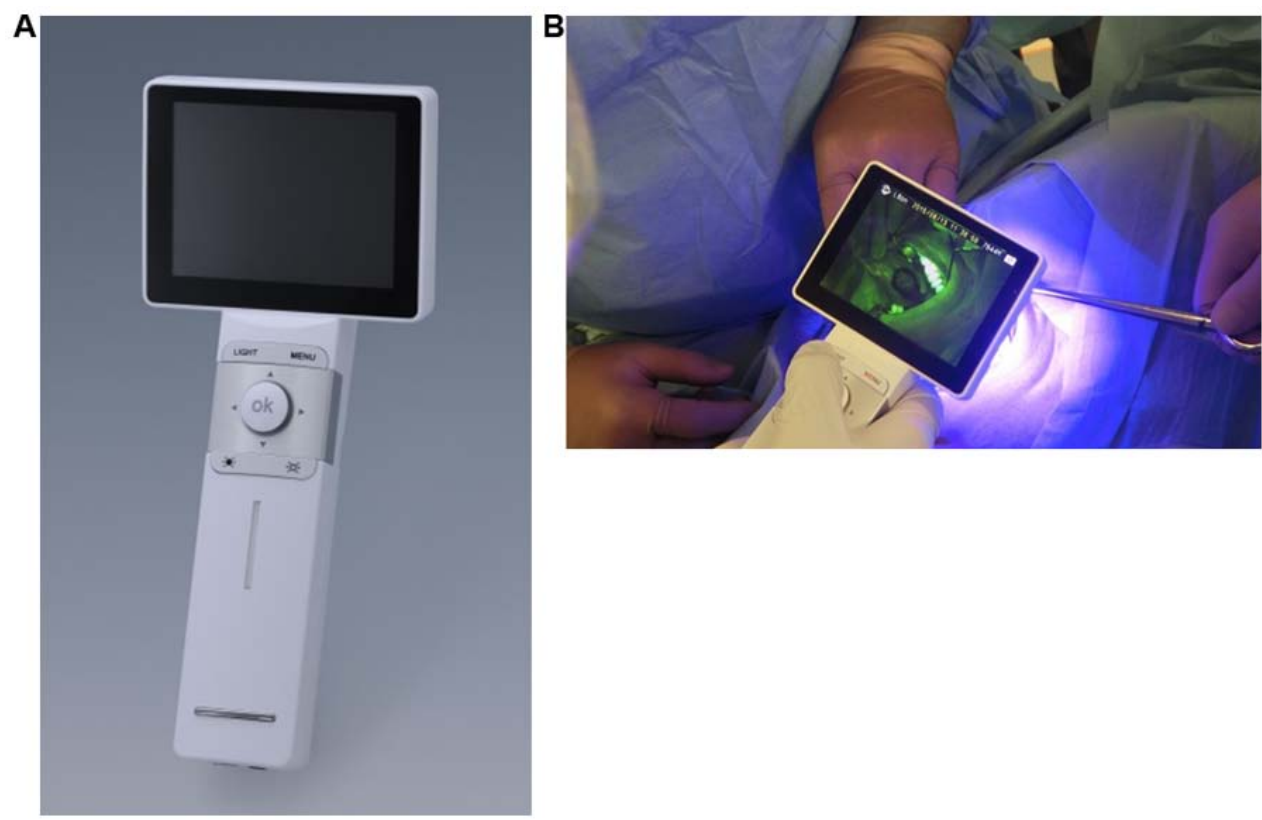

Figure 1. Device (IllumiScan ${ }^{\circledR}$ ). (A) Oral mucosa visualization device used in the present study. (B) Method used for fluorescence visualization.

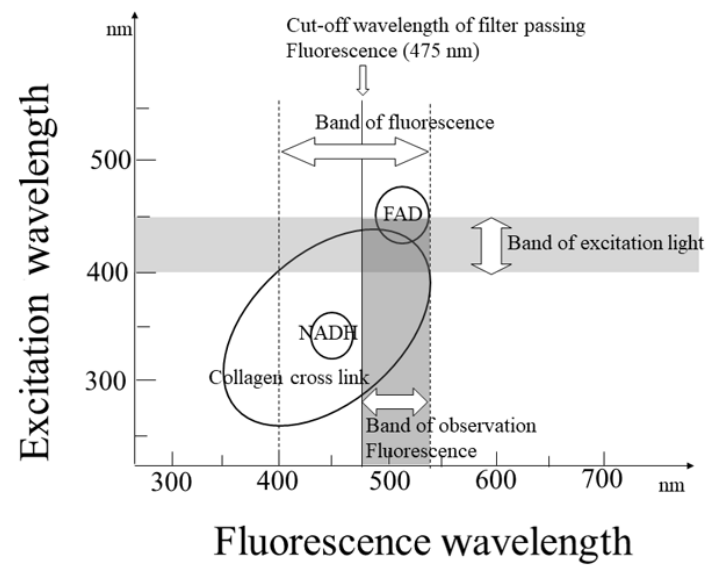

Figure 2. Principle of fluorescence visualization. Excitation light for irradiation and wavelength of the generated fluorescence. NADH, nicotinamide adenine dinucleotide; FAD, flavin adenine dinucleotide.

tissue and resultant tendency for more intensive fluorescence. Furthermore, for the determination of the extent of lesion resection, we compared an iodine-unstained area (identified by the conventional iodine staining method) to an image produced by the fluorescence visualization method described in the present study.

Measurement environment. The measurement of fluorescence was performed in a standardized clinical environment, where natural and artificial lighting could be blocked.

Resection method. Fluorescence light was irradiated onto the area of resection (resection range) of OSCC to mark the extent of FVL and the iodine-unstained area; the resection was performed under general anesthesia by considering the position that was $5 \mathrm{~mm}$ from the outermost circumference of FVL as the resection stump. The resection specimen was fixed in an extended position and marked to confirm the extent of FVL on the tissue specimen. Histopathological assessments were performed by hematoxylin and eosin (H\&E) and immunohistochemical staining (CK13, CK17, Ki67, p53, and E-cadherin) of the FVL(+) area and the resection stump FVL(-) as well as by Picro-Sirius Red staining to examine the CCL.

Histopathological assessment. Approximately 4-mm thick slices were prepared from the paraffin-embedded blocks of resected tissue, and one slice was subjected to H\&E staining, 


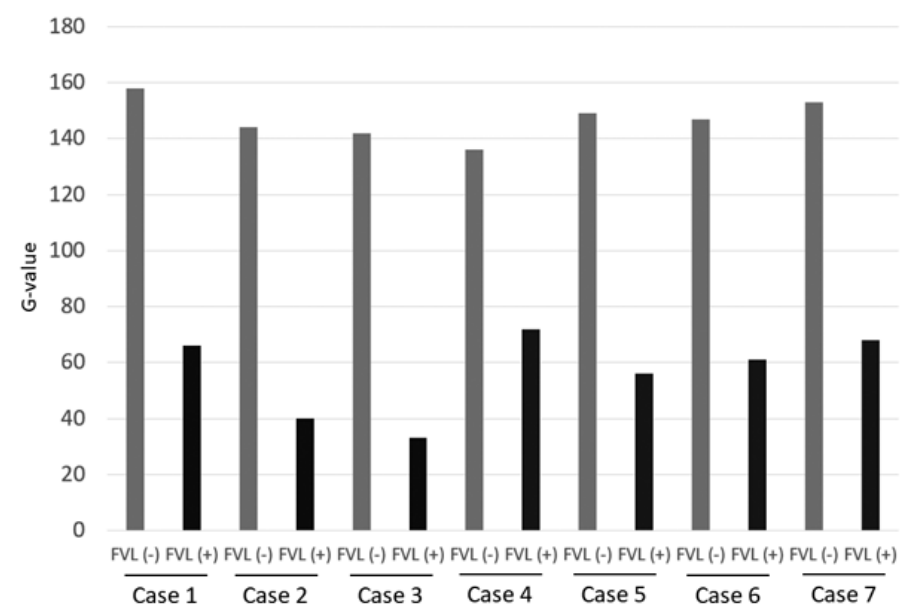

Figure 3. Results of fluorescence luminance in patients with oral mucosal disease. FVL(+), fluorescence visualization loss; FVL(-), no fluorescence visualization loss.

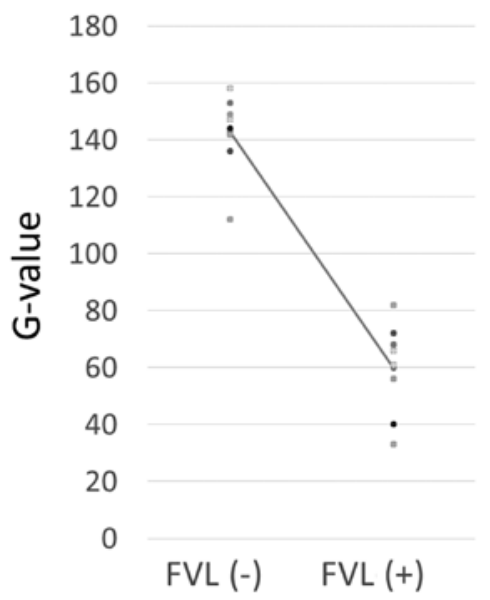

Figure 4. Comparison of G values for the FVL(+) and FVL(-) areas. There was a statistically significant difference between the two groups $(\mathrm{P}=0.0001)$. $\mathrm{FVL}(+)$, fluorescence visualization loss; FVL(-), no fluorescence visualization loss.

whereas the others were subjected to CK13, CK17, Ki67, p53, and E-cadherin staining as well as to Picro-Sirius Red staining. The following antibodies were employed: Monoclonal anti-CK13 (Dako, F7), monoclonal anti-CK17 (Dako, E3), monoclonal anti-Ki67 (Dako, clone MB-1), and monoclonal anti-p53 (Dako, clone p53). Processing was performed using an automatic stainer (Autostainer 48; Ventana). All the cases included basal oral epithelial cells, which showed nuclear positivity, as positive controls. In negative controls, the primary antibody was omitted. The percentage of positive nuclei in 400 consecutive epithelial cells in the selected areas representative of the lesion was determined and provided a semi-quantitative evaluation of the immunohistochemical results. The assessment method reported by Shiozaki et al (6) was used to evaluate the expression. The entire specimen was observed; samples with protein expression in the cell membrane similar to that in the FVL(-) area (the positive control) were regarded to be strongly accumulated, whereas samples that showed protein expression in an even, weak staining pattern were considered to be accumulated; no protein expression over the entire specimen was considered to be negative. Picro-Sirius
Red staining was performed by deparaffinizing the samples, incubating them with phosphomolybdic acid for $2 \mathrm{~min}$ and Picro-Sirius Red F3BA staining solution for $60 \mathrm{~min}$, and treating them with $0.1 \mathrm{~N}$ hydrochloric acid for $2 \mathrm{~min}$ and with $70 \%$ ethanol for $45 \mathrm{sec}$. The color of collagen was determined in the three categories as proposed by Aparna and Charu (7) and its density was evaluated. The assessments were performed by two pathologists.

Statistical analysis. The paired t-test was used to assess the statistical significance of differences between the samples. $\mathrm{P}<0.05$ was considered to indicate a statistically significant difference.

\section{Results}

Clinical information. This study included seven patients with squamous cell carcinoma of the tongue. There were five male and two female individuals, and the mean age of the subjects was 55 years (range, 43-76 years). On the basis of observation of the disease, two patients had granular tumors, two had diffuse tumors, two had a mix of vitiligo and diffuse tumors, and one patient had a vitiligo-type tumor. The detailed clinical information for all the patients is presented in Table I.

$G$ value measurements. The G values for the $\mathrm{FVL}(+)$ and FVL(-) areas in the tumors of each subject are shown Fig. 3. The $\mathrm{G}$ values for the FVL(+) area ranged between 33 and 72 , with a mean of 56, whereas those for the FVL(-) area were between 136 and 158, with a mean of 147. There were statistically significant differences between the two groups (Fig. 4). The fluorescence luminance $G$ was lower than 80 in the FVL(+) areas and higher than 130 in the FVL(-) areas for all the subjects (Fig. 3).

Histopathological assessment. The FVL(+) area showed destruction of collagen and roughness in the Picro-Sirius Red staining, whereas the FVL(-) area did not show any disruption of the collagen arrangement. However, collagen exhibited a slightly rough pattern in the FVL (+) areas in cases 3,4 and 7. All specimens showed category 1 (reddish-orange) birefringence. 
A

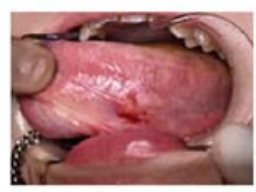

C

$\mathrm{H} \& \mathrm{E}$
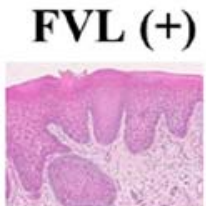

CK13

CK17

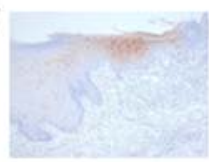

Ki67
B

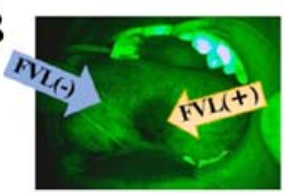

FVL (-)
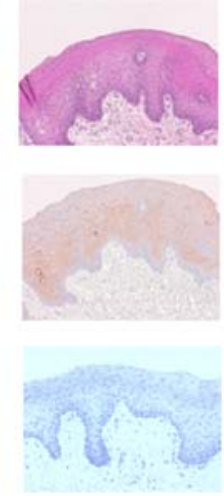

p53

E-cadherin

Picro-sirius Red

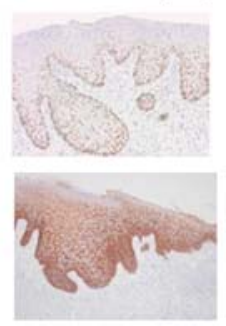

\section{【Case 1】 \\ $62 y$ male SCC}

FVL (+) FVL (-)
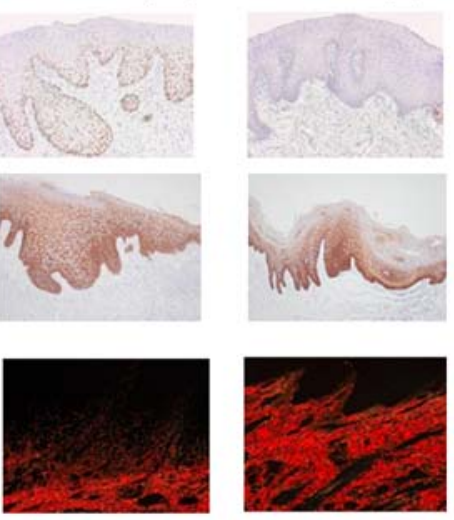

Figure 5. Left-sided tongue cancer in a 62-year-old male. (A) Image of the oral cavity. (B) Fluorescence visualization. (C) Histopathological assessment. H\&E, CK13, CK17, Ki67, p53 and E-cadherin staining (magnification, x40), and Picro-Sirius Red staining (magnification, x200). FVL(+), fluorescence visualization loss; FVL(-), no fluorescence visualization loss; H\&E, hematoxylin and eosin; SCC, squamous cell carcinoma.

A

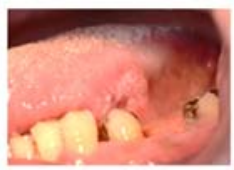

B

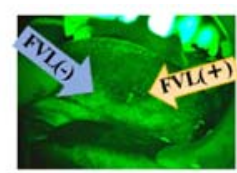

C

FVL (+)

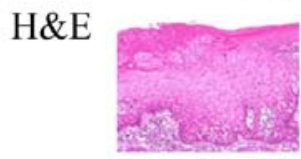

CK13

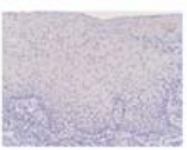

CK17

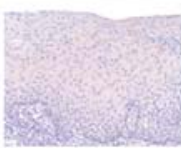

Ki67
FVL (-)
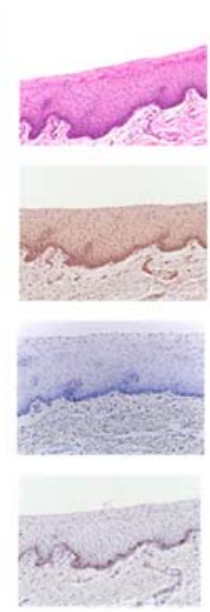

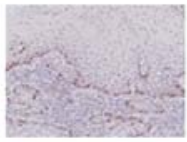

p53

E-cadherin

FVL (+)

FVL (-)
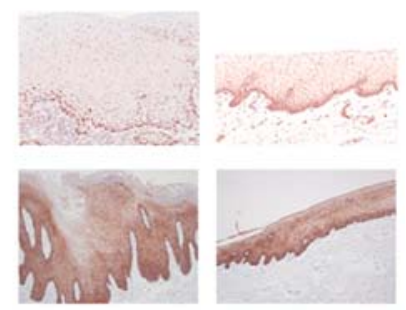

Picro-sirius

Red
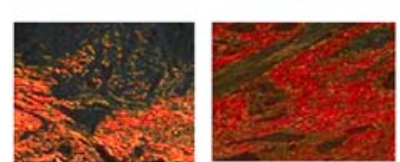

Figure 6. Left-sided tongue cancer in a 59-year-old male. (A) Image of the oral cavity. (B) Fluorescence visualization. (C) Histopathological assessment. H\&E, CK13, CK17, Ki67, p53 and E-cadherin staining (magnification, x40), and Picro-Sirius Red staining (magnification, x200). FVL(+), fluorescence visualization loss; FVL(-), no fluorescence visualization loss; H\&E, hematoxylin and eosin; SCC, squamous cell carcinoma.

Although there was pronounced CCL destruction, its extent varied for the different cases. The staining for CK13 was negative in the FVL(+) areas and positive in the FVL(-) areas, whereas the opposite was true for CK17. The expression of p53 was positive in the FVL(+) areas. The expression of Ki67 was positive throughout the entire epithelium for the FVL(+) areas and was only noted in the basal cell layer for the FVL(-) areas. Extensive staining was observed for E-cadherin in the FVL(-) 
area but was sparse in the FVL(+) area in all the subjects. The luminance $G$ value tended to be lower in the subjects with weaker E-cadherin staining in the FVL(+) area (Figs. 5 and 6; Table II).

\section{Discussion}

Oral squamous cell carcinoma is one of the common malignancies with significant morbidity and mortality. Its metastatic and invasive potential results in poor prognosis. Oral squamous cell carcinoma is often associated with loss of eating and speech functions, disfigurement, and psychological distress. The primary treatment for this disease is surgical intervention. Despite considerable advances in treatment over the past two decades, the overall disease outcome has improved only modestly (8). Local tumor recurrence affects approximately $60 \%$ of patients, and metastases develop in $15-25 \%$ (9). The prevention and management of oral squamous cell carcinoma will greatly benefit from the identification of molecular biomarkers and therapeutic targets for the disease $(10,11)$.

Many cases of OSCC occur in readily visualized areas, and the disease has, therefore, been regarded as easy to diagnose. In the past, the condition was clinically diagnosed only by visual observation and palpation followed by cytological and histological examination. However, a variety of pathological conditions of the oral mucosa, such as leukoplakia, oral lichen planus, oral candidiasis, and aphthous stomatitis, may be very difficult to differentiate from early-stage cancer simply by visual observation owing to similarities in their clinical presentation. In recent years, a simple and feasible vital staining by iodine and toluidine blue has been employed, which reportedly facilitates the disease staging and diagnosis of advanced epithelial dysplasia and early-stage cancer. However, this approach cannot be applied in all cases and in particular in outpatients due to restrictions, such as patient discomfort and allergies to the staining solutions (1).

However, the advancement of optical engineering in recent years has significantly contributed to the diagnosis and treatment of various oral mucosa diseases including oral cancer. Since 2008, researchers from North America have attempted to develop new methods based on optical approaches for the examination and diagnosis of oral cancer. Optical instruments, such as the magnifying endoscope $\mathrm{NBI}^{\circledR}$ (Narrow Band Imaging) system (12-14) and VELscope ${ }^{\circledR}$ (DenMat) (2-5), have been introduced for use in non-invasive and early discovery of OSCC. The NBI ${ }^{\circledR}$ system is an image enhancement technology that was jointly developed by Olympus Medical Systems and the Department of Gastroenterological Endoscopy, National Cancer Center Hospital East. It clearly visualizes the vascular structure and minute changes in the mucosal surface layer by irradiation with light in two narrow spectral widths of 415 and $540 \mathrm{~nm}$, through a narrow band filter, which is strongly absorbed by hemoglobin. As a result, the system clearly depicts the intraepithelial papillary capillary loop present in the superficial layer of the mucous membrane, thereby, serving as an indicator to diagnose malignancies, including esophageal and oral cancer. Because this system is expensive, large, and requires partial sterilization despite being a non-contact device, it is not easy to install, and its widespread deployment in dental clinics has been impeded. 
The portable optical instrument manufactured by Shofu Inc., which was used in the present study, irradiates the surface with a 425-nm blue light and enables the visualization of oral mucosal diseases. Using this instrument, an image analysis software that digitized the color of the mucous membrane ( $G$ value) was developed to obtain objective data that cannot be acquired with vital staining and conventional fluorescence visualization devices (5). As a result, minute changes in fluorescence that could not be recognized by visual inspection could be recorded in a digitized form, and color changes, otherwise unrecognizable by direct observation, could be detected by the software. Although the principle of fluorescence visualization is presumably related to the decrease in FAD levels and destruction of CCL, based on clinical experience with presented cases for which the results of fluorescence visualization did not match the pathology, we inferred that some other factor is also implicated. Therefore, in the present study, we focused on immunohistochemical staining of CK13, CK17, Ki67, p53, and E-cadherin, especially on the cell adhesion molecule, E-cadherin.

Cell adhesion molecules include members of the immunoglobulin superfamily, integrin superfamily, and cadherin family. Among them, E-cadherin, which plays a central role in the adhesion between epithelial cells, is expressed in epithelial tissues, and is a classic cadherin involved in the formation of adherence junctions. It binds to intracellular $\beta$ - and $\gamma$-catenin and to the cytoskeleton, including $\alpha$-catenin and actin filaments (15). In normal stratified squamous epithelium, E-cadherin is observed in the stratum basale and stratum spinosum, but not in the basal lamina. In recent years, cancer infiltration and metastases have been attributed to epithelial-mesenchymal transition (EMT) of epithelial tumor cells, which leads to the loss of their epithelial characteristics and migration through the stroma in a disseminated manner. E-cadherin has been used as an epithelial marker in the assessment of EMT, and its attenuation or disappearance is considered an indicator of complete EMT $(16,17)$. The reduced expression of the E-cadherin and catenin family members is observed in a variety of cancer types and is strongly associated with infiltration, metastasis, and prognosis $(18,19)$. Schipper et al (20) reported that the abnormal expression of E-cadherin in head and neck squamous cell carcinoma was related not only to the degree of cancer differentiation but also to the presence or absence of cervical lymph node metastases; furthermore, similar results were reported for OSCC (21). In these studies, approximately $50 \%$ of the subjects presented with attenuated E-cadherin expression. However, to the best of our knowledge, there are no reports on the relationship between E-cadherin expression and fluorescence visualization.

In the present study, the G value was higher in the FVL(-) areas than in the $\mathrm{FVL}(+)$ areas for all subjects for whom fluorescence visualization was conducted. Furthermore, the Picro-Sirius Red staining revealed no CCL destruction in the FVL(-) areas, whereas the extent of CCL destruction in the FVL(+) areas varied with the subject. CK13 expression was positive in normal oral mucosa and negative in intraepithelial tumors, whereas the opposite tended to be true for CK17 expression. All seven subjects in the present study showed the same results. Because there is a positive correlation between the expression level of Ki67 and tumor malignancy, it would be useful as a marker for the detection of proliferating cells in tumor tissues. Furthermore, p53 has a very short half-life in normal cells, and is not present in quantities that would result in positive immunostaining. E-cadherin was expressed extensively in the FVL(-) areas. In contrast, although the FVL(+) areas had lower G values than the FVL(-) areas in all the subjects, the expression of E-cadherin was attenuated regardless of the extent of CCL destruction. Furthermore, subjects with strong attenuation of E-cadherin expression tended to have low $\mathrm{G}$ values.

Even though the number of specimens examined in this study was small and relationship to local recurrence was not been studied, the results suggest the involvement of attenuation of E-cadherin expression regardless of the decrease in $G$ value and extent of CCL destruction as a factor contributing to the principle of fluorescence regulation, in addition to a decrease in FAD level and destruction of CCL. It is our ultimate goal to use this device for setting the surgical margins for oral squamous cell carcinoma. The present study describes a preliminary step in this direction. And we would need to conduct further studies on a larger number of patients to validate our results, particularly regarding the relationship between the histopathological appearances and the $\mathrm{G}$ value.

In conclusion, the results of this study suggest that a decrease in the luminance $G$ value in oral mucosal fluorescence visualization is related to the cell adhesion factor, E-cadherin, and that this may contribute to a new principle underlying the regulation of fluorescence from oral mucosa.

\section{Acknowledgements}

The authors would like to thank Dr Eiko Suzuki (Department of Oral Pathobiological Science and Surgery, Tokyo Dental College, Tokyo, Japan) for technical advice.

\section{Funding}

This research was supported by grants for Private University Branding Project supported by Ministry of Education, Culture, Sports, Science and Technology, Japan, and Tokyo Dental College Branding Project for Multidisciplinary Research Center for Jaw Disease (MRCJD): Achieving Longevity and Sustainability by Comprehensive Reconstruction of Oral and Maxillofacial functions.

\section{Availability of data and materials}

The datasets used and/or analyzed during the current study are available from the corresponding author on reasonable request.

\section{Authors' contributions}

KS and AK developed the study design, analyzed the data, and wrote the original manuscript. YK and MK analyzed the data. $\mathrm{SM}, \mathrm{KO}, \mathrm{KK}, \mathrm{KN}, \mathrm{KM}$ and $\mathrm{SA}$ contributed to data analysis, and manuscript writing and revision. All authors read and approved the final manuscript.

\section{Ethics approval and consent to participate}

The present study was conducted with the approval of the Tokyo Dental College Ethics Committee (approval no. 759). 
Informed consent was obtained from all the participants, and this informed consent included consent to participate in the study and consent to publish their images.

\section{Patient consent for publication}

Informed consent was obtained from all the participants, and this informed consent included consent to participate in the study and consent to publish their images.

\section{Competing interests}

The authors declare that they have no competing interests.

\section{References}

1. Takano M, Kakizawa T, Takasaki Y, Seta S, Noma H, Yajima Y and Nomura S: Clinical classification to indicate stage of oral precancerous lesions and early cancer with iodine and toluidine blue staining test. Head Neck Cancer 28: 41-46, 2002.

2. Lane $P(e d)$ : Fluorescence instrumentation for the direct visualization of oral mucosa. In: The Inside Summit on Oral Cancer Discovery and Management. The technologies and the Role of Dental Clinicians. Boston, 2017.

3. Poh CH, Zhang L, Anderson DW, Durham JS, Williams PM, Priddy RW, Berean KW, Ng S, Tseng OL, MacAulay C and Rosin MP: Fluorescence visualization detection of field alterations in tumor margins of oral cancer patients. Clin Cancer Res 12: 6716-6722, 2006.

4. Poh CF, Ng SP, Williams PM, Zhang L, Laronde DM, Lane P, Macaulay C and Rosin MP: Direct fluorescence visualization of clinically occult high-risk oral premalignant disease using a simple hand-held device. Head Neck 29: 71-76, 2007.

5. Sugahara K, Futoo E, Bessho H, Sekine R, Ohno K, Katakura A and Shibahara T: Fluorescence visualization for oral mucosa using an auto-fluorescence imaging analysis software. J Jap Soc Oral Diagn/Oral Med 30: 168-175, 2017.

6. Shiozaki H, Tahara H, Oka H, Miyata M, Kobayashi K, Tamura S, Iihara K, Doki Y, Hirano S, Takeichi M, et al: Expression of immunoreactive E-cadherin adhesion molecules in human cancer. Am J Pathol 139: 17-23, 1991.

7. Aparna V and Charu S: Evaluation of collagen in different grades of oral squamous cell carcinoma by using picrosirius red stain-A histochemical study. J Clin Diagn Res 4: 3444-3449, 2010.

8. Eheman C, Henley SJ, Ballard-Barbash R, Jacobs EJ, Schymura MJ, Noone AM, Pan L, Anderson RN, Fulton JE, Kohler BA, et al: Annual Report to the Nation on the status of cancer, 1975-2008, featuring cancers associated with excess weight and lack of sufficient physical activity. Cancer 118 2338-2366, 2012.
9. Genden EM, Ferlito A, Bradley PJ, Rinaldo A and Scully C: Neck disease and distant metastases. Oral Oncol 39: 207-212, 2003.

10. Sabichi AL, Demierre MF, Hawk ET, Lerman CE and Lippman SM: Frontiers in cancer prevention research. Cancer Res 63: 5649-5655, 2003.

11. Spafford MF, Koch WM, Reed AL, Califano JA, Xu LH, Eisenberger CF, Yip L, Leong PL, Wu L, Liu SX, et al: Detection of head and neck squamous cell carcinoma among exfoliated oral mucosal cells by microsatellite analysis. Clin Cancer Res 7: 607-612, 2001.

12. Inoue H, Honda T, Nagai K, Kawano T, Yoshino K, Takeshita K and Endo M: Ultra-high magnification endoscopic observation of carcinoma in situ of the esophagus. Dig Endosc 9: 16-18, 1997.

13. Takano JH, Yakushiji T, Kamiyama I, Nomura T, Katakura A, Takano N and Shibahara T: Detecting early oral cancer: Narrowband imaging system observation of the oral mucosa microvasculature. Int J Oral Maxillofac Surg 39: 208-213, 2010.

14. Sekine R, Yakushiji T, Tanaka Y and Shibahara T: A study on the intrapapillary capillary loop detected by narrow band imaging system in early oral squamous cell carcinoma. J Oral Maxillofac Surg 27: 624-630, 2015.

15. Takeichi M: Cadherin cell adhesion receptors as a morphogenetic regulator. Science 251: 1451-1455, 1991.

16. Thiery JP: Epithelial-mesenchymal transitions in tumour progression. Nat Rev Cancer 2: 442-54, 2002.

17. Thiery JP and Sleeman JP: Complex networks orchestrate epithelial-mesenchymal transitions. Nat Rev Mol Cell Biol 7: 131-142, 2006.

18. Ma L, Liu L, Ma Y, Xie H, Yu X, Wang X, Fan A, Ge D, Xu Y, Zhang $Q$ and Song C: The role of E-cadherin/ $\beta$-catenin in hydroxysafflor yellow A inhibiting adhesion, invasion, migration and lung metastasis of hepatoma cells. Biol Pharm Bull 40: 1706-1715, 2017.

19. Shiozaki H, Doki Y, Kawanishi K, Shamma A, Yano M, Inoue M and Monden M: Clinical application of malignancy potential grading as a prognostic factor of human esophageal cancers. Surgery 127: 552-561, 2000.

20. Schipper JH, Frixen UH, Behrens J, Unger A, Jahnke K and Birchmeier W: E-cadherin expression in squamous cell carcinomas of head and neck: Inverse correlation with tumor dedifferentiation and lymph node metastasis. Cancer Res 51: 6328-6337, 1991.

21. Schipper JH, Unger A and Jahnke K: E-cadherin as a functional marker of the differentiation and invasiveness of squamous cell carcinoma of the head and neck. Clin Otolaryngol Allied Sci 19: 381-384, 1994.

This work is licensed under a Creative Commons Attribution-NonCommercial-NoDerivatives 4.0 International (CC BY-NC-ND 4.0) License. 